\title{
The Kyoto 3-D Spectrograph and an Imaging Fabry-Perot Observation of the Nuclear Region of NGC 4151
}

\author{
H. Ohtani, T. Ishigaki, T. Hayashi, S. Ozaki, and T. Hattori \\ Department of Astronomy, Kyoto University, Kyoto 606-01, Japan \\ M. Sasaki \\ Shimonoseki City University, Shimonoseki 751, Japan \\ K. Aoki \\ National Astronomical Observatory, Mitaka 181, Japan \\ M. Yoshida and E. Watanabe \\ Okayama Astrophysical Observatory, Kamogata 719-02, Japan
}

\section{The Kyoto 3-D Spectrograph}

The Kyoto 3-D Spectrograph was commissioned successfully at the $188-\mathrm{cm}$ telescope of the Okayama Astrophysical Observatory in the spring of 1996. This instrument has four distinct modes (Ohtani et al. 1994): (1) narrow-band imager, which is an ordinary focal-reducer camera; (2) Spectro-NebulaGraph (long-slit spectrograph; Kosugi et al. 1995); (3) imaging Fabry-Perot interferometer, using either of two Fabry-Perot etalons from Queensgate Instruments (a tunable filter with $R=300$ and another with $R=7000$ for velocity-field observations. Broad-band (400-700 nm) coatings are deposited on both etalons. During observations, the etalon temperature is stabilized within $0.5^{\circ} \mathrm{C}$ ); and (4) integral-field spectrograph of the TIGER-type (Bacon et al. 1995). In this mode, the spectra of $7 \times 11$ objects can be recorded simultaneously, along with $7 \times 2$ spectra of the sky $4^{\prime}$ away. The spatial resolution is $1^{\prime \prime} .3$ and the field of view is $9^{\prime \prime} \times 14^{\prime \prime}$.

\section{Observations of NGC 4151}

Here we give the first results of the imaging Fabry-Perot interferometric observations. The velocity field of the central regions of the extended emission-line region of the Seyfert galaxy NGC 4151 was observed in the [O III] $\lambda 5007$ line with the higher-resolution etalon. Photometric corrections for variations of the atmospheric transmission during scans were made from the variations of the brightness of the guide star monitored by the off-set guider.

The velocity field and the distribution of the line width in a $10^{\prime \prime}$ field around the nucleus was derived. In Fig. 1a, the line-width distribution is shown in a grey scale together with the contour map of the velocity field. It is apparent that the regions of the larger line widths extend biconically from the nucleus. The shape and the orientation of the cones are very similar to the biconical structure 

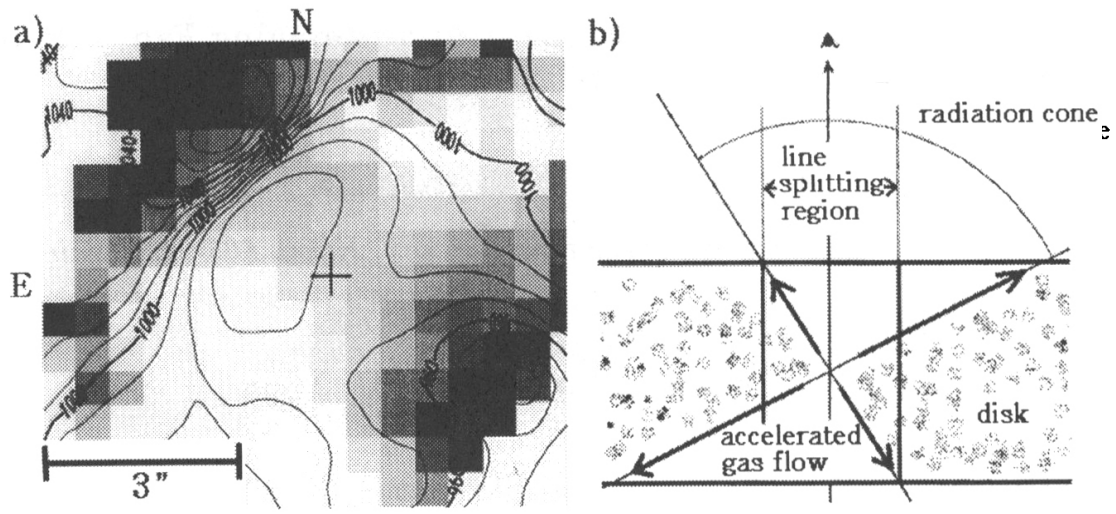

Figure 1. (a) The velocity field (contours) and the distribution of the velocity dispersion (grey scale) of the ionized gas around the nucleus (cross). (b) An interpretation of the observational results.

of the extended ionized gas. Line splitting is seen in the NE region, consistent with the result of Schulz (1990). In the contour map, a large velocity gradient exists $2^{\prime \prime} \mathrm{NE}$ from the nucleus, as is recognized in the velocity curve of Robinson et al. (1994). In our data, the $750 \mathrm{~km} \mathrm{~s}^{-1}$ component SW of the nucleus (Schulz 1990 , Ulrich 1973) is missed; this may be due to contamination by the scattered light from the nucleus or to errors in the spatial registration procedure of frames of different etalon gaps. The line widths increase with distance from the nucleus and suddenly decrease at $3^{\prime \prime} \mathrm{NE}$ and $3^{\prime \prime} \mathrm{SW}$.

These results may be interpreted as illustrated in Fig. 1b. We are observing mainly ionized gas outflowing with accelerations along the surfaces of the biconical radiation cones. At the edges made by the intersection of the galactic disk surfaces with the radiation cone of the near side, a sudden diminishing of the bluer components of the line width occurs. On the further side, the opposite occurs.

\section{References}

Bacon, R., et al. 1995, A\&AS, 113, 347.

Kosugi, G., et al. 1995, PASP, 711, 475.

Ohtani, H., et al. 1994, in Instrumentaion in Astronomy VIII, Proc. SPIE Symp. Vol. 2198, 229.

Robinson, A., et al., 1994, A\&A, 291, 351.

Schulz, H. 1990, AJ, 99, 1442.

Ulrich, M. 1973, ApJ, 181, 51. 